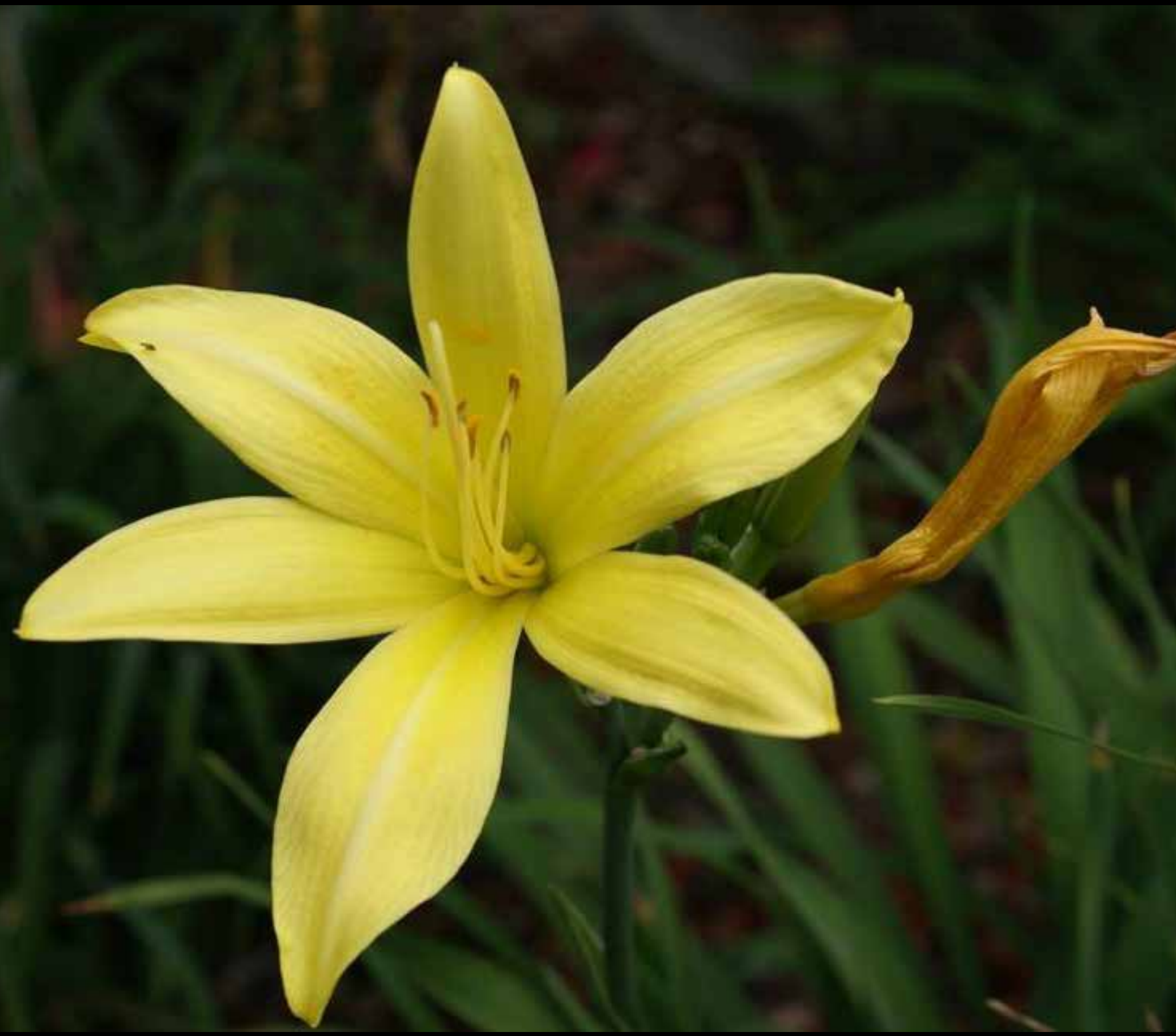




\title{
CONOCIMIENTO DIDÁCTICO DEL CONTENIDO DE UN PROFESOR DE BIOLOGÍA SOBRE EL CONCEPTO DE CÉLULA: IMPLICACIONES PARA LA ENSEÑANZA DE LA BIOLOGÍA
}

\section{Pedagogical Content Knowledge of a Biology Teacher about the Concept of Cell: Implications for Biology Teaching}

\section{Conhecimento Didático do Conteúdo de um Professor de Biologia sobre o Conceito de Célula: Implicações para o Ensino da Biologia}

\author{
Humberto Bohórquez-Salazar* \\ Édison Fernando Marín** \\ Cristian Camilo Torres-Niño ${ }^{* * *}$ \\ Jairo Robles-Piñeros ${ }^{\star \star \star \star}$
}

Fecha de recepción: 18 de febrero de 2019

Fecha de aprobación: 29 de abril de 2019

\section{Resumen}

En este artículo de investigación se desarrolla un análisis de componentes propios del Conocimiento Didáctico del Contenido (CDC) - siguiendo el modelo hexagonal propuesto por Park y Oliver (2008) - construidos por un docente de biología de grado sexto durante el proceso de enseñanza del concepto célula. Los datos fueron obtenidos mediante observación de clases, la aplicación de un cuestionario de representación del contenido (ReCo) y la revisión de los planes de área y de clase elaborados por el docente. El análisis se llevó a cabo a partir de tres categorías específicas del CDC: 1) orientaciones hacia la enseñanza de las ciencias, 2) el conocimiento sobre el currículo de las ciencias y 3) el conocimiento sobre la evaluación en ciencias. Se encuentra que el docente tiene una gran influencia de conocimientos teórico-conceptuales, los cuales hacen que vea la biología como una ciencia acumulativa de conceptos inalterados, manteniéndola en un paradigma empírico-positivista, lo que hace que vea la enseñanza de la misma como una mera transmisión de contenidos. Se hace evidente la importancia del CDC como punto de quiebre para la enseñanza de las ciencias y sus implicaciones sobre cómo, desde la formación pedagógica impartida en las licenciaturas se podría llegar a un desarrollo más amplio de estos componentes.

Palabras clave: Conocimiento Didáctico del Contenido; célula; didáctica de la biología; enseñanza de la biología; formación de profesores de ciencias

* Magister en Educación, Universidad Pedagógica y Tecnológica de Colombia. Docente Institución Educativa Santa Barbara - San Pablo de Borbur- Boyacá, Colombia. Codigo ORCID: https://orcid.org/0000-0002-2191-8086. Correo electrónico: humberto.bohorquez@uptc.edu.co

* Estudiante de Maestría en Educación, Universidad Pedagógica y Tecnológica de Colombia. Docente de Ciencias Naturales/ Colegio Los Ángeles-Tunja, Colombia. Código ORCID: 0000-0001-9976-7276. Correo electrónico: edinson.marin@uptc.edu.co

*** Estudiante de Maestría en Educación, Universidad Pedagógica y Tecnológica de Colombia. Código ORCID: https://orcid.org/0000-00026348-0334. Correo electrónico: cristiancamilo.torres@uptc.edu.co

**** Doctorado en Enseñanza, Filosofía e Historia de las Ciencias, Universidad Federal de Bahía / Universidad Estadual de Feira de Santana y Doctorado Interinstitucional en Educación (DIE-UD), Universidad Distrital Francisco José de Caldas. Código ORCID: https://orcid.org/00000002-8403-3066. Correo electrónico: jairo.robles@ufba.br 


\section{Abstract}

An analysis of components from Pedagogical Content Knowledge (PCK) is developed, following the Hexagonal Model from Park and Oliver (2008), built by a biology teacher of sixth grade during the process of teaching the concept of the cell. The data were obtained through the class observation, application of a content representation questionnaire (ReCo, in Spanish), and the review of the lesson planning elaborated by the teacher. The analysis was carried starting from three pск specific categories: 1) orientations toward the science teaching, 2) knowledge about science curriculum and 3) knowledge about the evaluation in science. The teacher has a significant influence of theoretical-conceptual knowledge, which makes him see biology as an accumulative science of unaltered concepts, maintaining it into an empirical-positivist paradigm, what makes it see the teaching of it as a mere transmission of content. The importance of the PсK as a breaking point for the teaching of science and its implications on how - from the pedagogical training given in the bachelor degrees-could reach a more extensive development of these components is evident.

Keywords: biology teaching; cell; didactics of Biology; Pedagogical Content Knowledge; science teacher training

\section{Resumo}

Desenvolve-se uma análise dos componentes próprios do Conhecimento Didático do Conteúdo (CDC), seguindo o modelo hexagonal de Park e Oliver (2008), construídos por um docente de biologia do sexto ano durante o processo de ensino do conceito célula. Os dados foram obtidos através de observação de aulas, a aplicação de um questionário de representação do conteúdo (ReCo) e a revisão dos planos de aula elaborados pelo docente. $O$ analise foi desenvolvido a partir de três categorias específicas do CDC: 1) orientações ao ensino das ciências, 2) o conhecimento sobre o currículo de ciências e 3) o conhecimento sobre a avaliação em ciências. Observa-se que o docente tem uma grande influência de conhecimentos teórico-conceituais, os quais fazem com que ele veja a biologia como uma ciência acumulativa de conceitos inalterados mantendo-a dentro de um paradigma empírico-positivista, o que faz que enxergue o ensino da mesma como uma mera transmissão de conteúdo. Se faz evidente a importância do cDc como ponto de quebre para o ensino das ciências e suas implicações sobre como, a partir da formação pedagógica ministrada nas licenciaturas poderia chegar num desenvolvimento mais amplo destes componentes.

Palavras-chave: Conhecimento Didático do Conteúdo; célula; didática da biologia; ensino da biologia; formação de professores de ciências 


\section{Introducción}

Para Gallego y Gallego (2006), la enseñanza es la acción o el efecto de enseñar y supone dos componentes: quien recibe la enseñanza (aprendiz, estudiante, alumno, educando, etc.) y la persona que enseña (maestro, docente, profesor, etc.). En la mayoría de los casos, este último es un profesional formado desde una disciplina específica (matemáticas, idiomas, ciencias sociales, ciencias naturales, etc.), con unos principios pedagógicos y otros didácticos (generales y específicos). Estos componentes (estudiante-docente) se han relacionado, por tradición, desde un enfoque mecanicista e individualista, en el cual el segundo se dedica únicamente a instruir y transmitir de manera expositiva un conjunto de saberes, conocimientos o contenidos considerados de carácter científico (de Jong y Van der Valk, 2007), los cuales son vistos como los únicos representantes del conocimiento en las aulas de clase. Adicionalmente, a los estudiantes les corresponde un papel de pasividad, de escucha o de simple retención y transmisión de esos saberes en el proceso evaluativo.

Esta visión mecanicista y transmisiva de la enseñanza implica, por un lado, un problema de fragmentación de los conocimientos que se enseñan, así como de su naturaleza (Adúriz-Bravo, 2005a) y, por otro, un problema general en el propio proceso de enseñanza (Cossío y Hernández, 2016). Lo anterior implica que, al menos en el campo de las ciencias de la naturaleza, la enseñanza tiene que adoptar como uno de sus objetivos prioritarios, el de ayudar a los alumnos a aprendery a enseñar ciencia, o, en otras palabras, enseñar a los alumnos procedimientos para el aprendizaje de las ciencias. No es que hasta ahora esos procedimientos estuvieran fuera de las aulas de clase, sino que en la mayoría de los casos no recibían un tratamiento didáctico específico (Pozo y Gómez, 2009). Como consecuencia de lo anterior se generó, en primera medida, un desconocimiento de que tanto estudiantes como profesores poseen un conjunto de concepciones específicas (conocimiento) sobre el mundo, las cuales funcionan como herramientas que permiten interpretar la realidad natural y luego crear explicaciones, para así manejarse dentro de ella; en segundo lugar, se olvida la existencia de obstáculos epistemológicos, que impiden adoptar perspectivas de acciones diferentes. El conocimiento científico se transmitía en su complejidad sin dar lugar a procesos de reflexión sobre su enseñanza ni sobre el contenido mismo (Pereira, 2010).

Dada la dinámica en la cual se encaminaba el proceso de la enseñanza de las ciencias, parecía ser claro que esta no podía seguirse realizando por transmisión oral de contenidos curriculares, ni el aprendizaje continuar siendo redu- cido al memorístico y al pie de la letra (Gallego y Gallego, 2006). Desde este punto de vista, las didácticas de las ciencias tenían que ser apartadas del modelo comeniano (partidarios de una didáctica general), de la concepción empírico-positivista y de la reducción algorítmica, incluyendo el método científico al que había estado sometida, sin posibilidades de constituirse como una disciplina conceptual y con soporte metodológico.

Es aquí donde los fundamentos epistemológicos planteados por diferentes autores como Bachelard, Kuhn, Lakatos, Feyerabend y Toulmin, y que han sido recopiladas en el trabajo de Lafrancesco (2005), proponen un rechazo hacia el modelo de transmisión-asimilación. Se pone de manifiesto la necesidad de modificar este paradigma, pretendiendo así que se deje de lado la reproducción memorística de conocimientos por parte de enseñante y alumnado. Sin embargo, no es suficiente con que se reflexione sobre los procesos de enseñanza y aprendizaje, o sobre el papel del docente y del alumno en estos procesos, hay que poner en el centro de la reflexión la naturaleza y el origen del conocimiento mismo, así como también comprender cuáles son los medios de su circulación y las formas de su apropiación (Zambrano, 2005). Dicho de otro modo, la preocupación actual no debe limitarse a estudiar el cómo enseñan los maestros, o en el cómo aprenden los estudiantes, se hace necesario ampliar el espectro de investigación y conocer qué es lo que enseñan los profesores y qué es lo que aprenden los alumnos, del mismo modo es necesario hallar respuestas a cómo se construye y transforma este conocimiento a lo largo del proceso.

Es así, como a partir de estas preocupaciones por la naturaleza y origen del conocimiento, de las relaciones explícitas e implícitas entre docentes y estudiantes, los acercamientos e interpretaciones de ellos frente al conocimiento, se tendría una aproximación más acertada a la hora de pensar, comprender y ejecutar tanto el proceso de enseñanza de las ciencias naturales, como de fortalecer los procesos de aprendizaje de estas (Friedrichsen, Driel y Abell, 2011). Lo anterior solo es posible de lograr, al menos en gran medida, a partir de investigaciones que conduzcan al análisis y reflexión de las prácticas docentes en ciencias naturales, ya que de esta manera es posible hacer una aproximación a la generación de cambios actitudinales y epistémicos importantes que fortalezcan el ser, el saber y el actuar de los docentes. De igual forma, dichas reflexiones permiten una evaluación y resignificación sobre su saber, lo cual conlleva el reconocimiento del docente, no solo como un participante activo en los procesos de enseñanza y de aprendizaje, sino como un sujeto epistemológicamente diferenciado, que construye un conocimiento propio, el cual permite trascender de la 
perspectiva técnica de la enseñanza a un nivel académico de producción de conocimiento sobre la disciplina científica y sobre la enseñanza de la misma (Martínez, 2016).

\section{El CDC-PCK': una aproximación a la naturaleza del saber docente}

Para comprender el carácter de las preocupaciones sobre el conocimiento de los docentes es necesario mencionar dos perspectivas que se centraron en ofrecer una respuesta acerca de la naturaleza del saber básico para la enseñanza, en otras palabras, el conocimiento propio del docente. La primera de estas es la idea de la transposición didáctica, iniciada con los trabajos de Michel Verret y que cobra mayor relevancia con las investigaciones de Yves Chevallard, dicha postura establece que dentro de toda relación didáctica, debe existir una preocupación relacionada con el saber. Este debe pasar de ser un saber sabio a ser un saber enseñado, sin perder de vista la distancia que los separa, hay de esta manera una transposición didáctica, al menos en una dimensión sensu lato ${ }^{2}$ (Gómez, 2005). La otra perspectiva surge de los trabajos de Lee Shulman, a partir de la necesidad de establecer un conocimiento base para la enseñanza, se puede decir que Shulman va más allá de la idea de transposición didáctica y plantea la idea de un Conocimiento Didácticodel Contenido (CDC) el cual emerge como puente para la enseñanza de un saber específico y como fuente de conocimiento propio de los profesores, además se configura como una amalgama entre el conocimiento del contenido y el conocimiento didáctico (Bolívar, 2005; Can, Doğru y Bayir, 2017; Park, Jang, Chen y Jung, 2011), que permite al profesor representar, organizary adaptar un contenido para su enseñanza.

Las propuestas iniciales de Shulman $(1986,1987)$, buscaron dar soluciones a cuestionamientos como: ¿Cuáles son las fuentes de conocimiento del profesor? ¿Cómo decide el profesor qué enseñar? ¿Cuáles son sus fuentes de analogías, metáforas, ejemplos, demostraciones y expresiones? De esta forma, Shulman (1987) logró establecer una serie de categorías que permitieron conocer qué es lo que deben saber los profesores y sobre todo cómo logran adquirir o construir este saber. A partir de sus trabajos, identificó siete aspectos que constituyen el conocimiento base para la enseñanza en cada profesor, entendido este como aquel cuerpo de comprensiones, conocimientos,

2 Chevallard distingue dos "tipos" de transposición didáctica: una strictu sensu, la cual hace referencia al paso de un contenido o saber preciso a una versión didáctica nueva de este objeto de saber; y la dimensión sensu lato, que es el paso de un objeto de saber a un objeto de enseñanza, mediado por un estado de objeto de enseñar. habilidades y disposiciones que un profesor necesita para enseñar de modo efectivo en una situación dada, sin tener en cuenta su disciplina o materia (Grossman, Wilson y Shulman, 2005).

\section{Dichos aspectos son:}

1) El conocimiento del contenido, conocimiento disciplinar o conocimiento de la materia.

2) El conocimiento pedagógico general, el cual hace referencia a aquellos principios amplios y generales, estrategias de organización y gestión de los espacios escolares, y que parecen ser objetos de interés del proceso educativo.

3) El conocimiento del currículo, el cual está relacionado con la organización de los contenidos, los programas e identificación de los materiales propicios para la enseñanza de su materia.

4) El conocimiento del contexto educativo, el cual se centra en comprender el funcionamiento interno y externo del aula de clases, así como las características de las comunidades y culturas que tienen relación con el sistema educativo.

5) Un conocimiento sobre las características del alumnado, aspectos cognitivos y motivaciones.

6) Un conocimiento sobre los fines, propósitos, objetivos y valores de la educación, a partir de los componentes históricos y filosóficos de la misma.

Y, por último, el aporte fundamental de los trabajos de Shulman $(1986,1987)$ :

7) Un conocimiento didáctico del contenido (CDC) el cual encierra el conocimiento de las representaciones, de las dificultades en el aprendizaje y de las estrategias para superarlas (Hashweh, 2005). Asimismo, está compuesto por lo que un maestro sabe, lo que hace y las razones de su acción (Park y Oliver, 2008).

A partir de lo anterior el CDC emerge como el saber propio de los profesores, pues su naturaleza implica que debe existir una movilidad intencional del conocimiento dentro del aula de clase, para que los aspectos de la enseñanza y la preparación, la identificación de ambientes propios para el aprendizaje y las responsabilidades que implica ser docente se conviertan en un saber profesional, el cual terminará constituyendo la esencia misma del maestro y del proceso de enseñanza (Ravanal, 2012). De igual modo, se configuraría como un saber epistemológicamente diferenciado, de carácter práctico, ya que surge y es vali- 
dado por la acción educativa tanto en el desarrollo de la práctica profesional, como desde el trabajo cotidiano y el conocimiento del contexto (Campos, 2016; Helmes y Strokes, 2013). Se manifiesta como rutinas o guiones de acción, como hábitos o habilidades y es construido por la integración de una gran cantidad de saberes provenientes de diversas fuentes (Martínez y Valbuena, 2013; Porlán, Rivero y Martín, 1997; Shulman, 1987); así como a partir de una reflexión en y sobre la práctica misma (Park y Oliver, 2008; Valbuena, Gutiérrez, Correa y Amórtegui, 2009) o sobre la de otros. Sin embargo, existen algunas posturas que consideran que este conocimiento no se puede estudiar y que en gran medida no es posible descubrir, debe ser construido de manera progresiva, partiendo de unas concepciones personales y de un constante ir y venir entre la teoría y la práctica integrando procesos de investigación (Rodríguez y Martínez, 2016).

\section{Modelos de CDC-PCK: una aproximación a su desarrollo}

Es necesario reconocer que, como cualquier otro constructo surgido, bien sea de la actividad reflexiva como de la acción investigativa, el CDC trae consigo un desarrollo conceptual bien diferenciado, discutido y soportado por un gran número de investigaciones. Dicho desarrollo puede entenderse desde al análisis y comprensión de varios periodos marcados por fuertes tendencias investigativas. Autores como Fonseca (2017a) reconocen la existencia de dos momentos relevantes en la conceptualización del CDC, el primero enmarcado en las consideraciones iniciales de Shulman y sus colaboradores (1987) y el segundo, a partir de los trabajos derivados de la cumbre sobre conocimiento didáctico del contenido realizada en Estados Unidos, en el año 2012. Sin embargo, esta distinción de los periodos de desarrollo va a depender mucho de la propia comprensión y perspectiva del autor. Por ejemplo, Hashweh (2013) divide el desarrollo de la conceptualización del CDC en tres momentos: el primero (al igual que Fonseca), a partir de la conceptualización inicial de Shulman $(1986,1987)$; un segundo momento, enmarcado en lo que él denomina un desarrollo tardío del CDC, que comprende las investigaciones llevadas a cabo entre los años 1980 y 1990; por último, el momento caracterizado por investigaciones en la especificidad del CDC, etapa comprendida entre finales de la década de los años noventa hasta la actualidad ${ }^{3}$.

3 Momento que puede enmarcarse con la publicación del libro Re-Examining Pedagogical Content Knowledge in Science Education de Berry, Friedrichsen y Loughran (2015), citado por Fonseca (2017a), así como de los artículos de investigaciones sobre CDC-PCK publicados posteriormente.
Si bien cada momento trajo consigo un gran número de modelos para explorar el CDC, estos no se alejan mucho de las consideraciones iniciales de Shulman, y se acompañan de nuevas formas de pensar y de conceptualizar el cDC, logrando aportar elementos específicos para comprender su construcción en la práctica docente, así como categorías para realizar su evaluación. A continuación, se muestran los aspectos fundamentales de los modelos más usados en las investigaciones sobre el CDC; se toman como referencia los trabajos teóricos de Fernández (2014), Fernández (2015) y Fonseca (2017a), y se hace un especial énfasis en el modelo hexagonal de conocimiento didáctico del contenido para enseñanza de las ciencias de Park y Oliver (2008), dada su relevancia en esta investigación.

Grossman (1990) establece que los profesores necesitan poseer un cuerpo de conocimientos profesionales, el cual engloba tanto los conocimientos de los principios pedagógicos generales como el conocimiento de la materia que será enseñada; al mismo tiempo, sistematiza los componentes del conocimiento base de los profesores y propone que este conocimiento está caracterizado por cuatro dominios, entendidos estos como cada conocimiento que requiere el profesor para su enseñanza. El CDC es el resultado de la transformación del conocimiento pedagógico, del contexto y del contenido específico. Por su parte, Carlsen (1999) amplía este espectro de dominios y divide el conocimiento del contexto en uno contextual específico, centrado en el conocimiento de los estudiantes y en la estructura del salón de clases; y un conocimiento contextual general, relacionado con aspectos de la nación, el estado, la comunidad y la escuela.

Al mismo tiempo, Magnusson, Krajciky Borko (1999) enfatizan en la importancia del CDC dentro de la enseñanza de las ciencias. Su modelo mantiene la esencia de los postulados de Grossman, pero integra un conocimiento sobre la evaluación; modifican el componente de "concepciones de los propósitos para enseñar el contenido" por "orientaciones para la enseñanza de la ciencia", siendo este el componente principal del CDC.

Por último, Gess-Newsome (1999) propone dos modelos teóricos para entender el CDC: el primero es un modelo integrativo, el cual considera que el CDC es una intersección entre los conocimientos pedagógicos más generales, el conocimiento del contexto y el conocimiento disciplinar de un contenido específico. Según esta visión, enseñar sería un acto de integrar el conocimiento propio de estos tres dominios y no es necesario que cada dominio se desarrolle a la par del otro, pueden construirse por separado, pero deben integrarse en el proceso de enseñanza. 
Además el modelo transformativo del CDC propone que este se da a partir de una transformación de los mismos dominios, de esta forma, el CDC se constituirá como un nuevo conocimiento que traería impacto en la práctica docente, este modelo no se preocupa por el desarrollo de los dominios base, pero sí en cómo estos se transforman durante la práctica.
Algunos años después, Park y Oliver (2008) construirían su modelo a partir de una modificación del de Magnuson, integran un componente relacionado con la eficacia del profesor, que ordenaría al docente reflexionar sobre qué es lo más eficaz en el aula, a partir de lo que él percibe de sus estudiantes. Al integrar este componente, surge el modelo hexagonal del conocimiento didáctico del contenido para la enseñanza de las ciencias (véase figura 1 ).

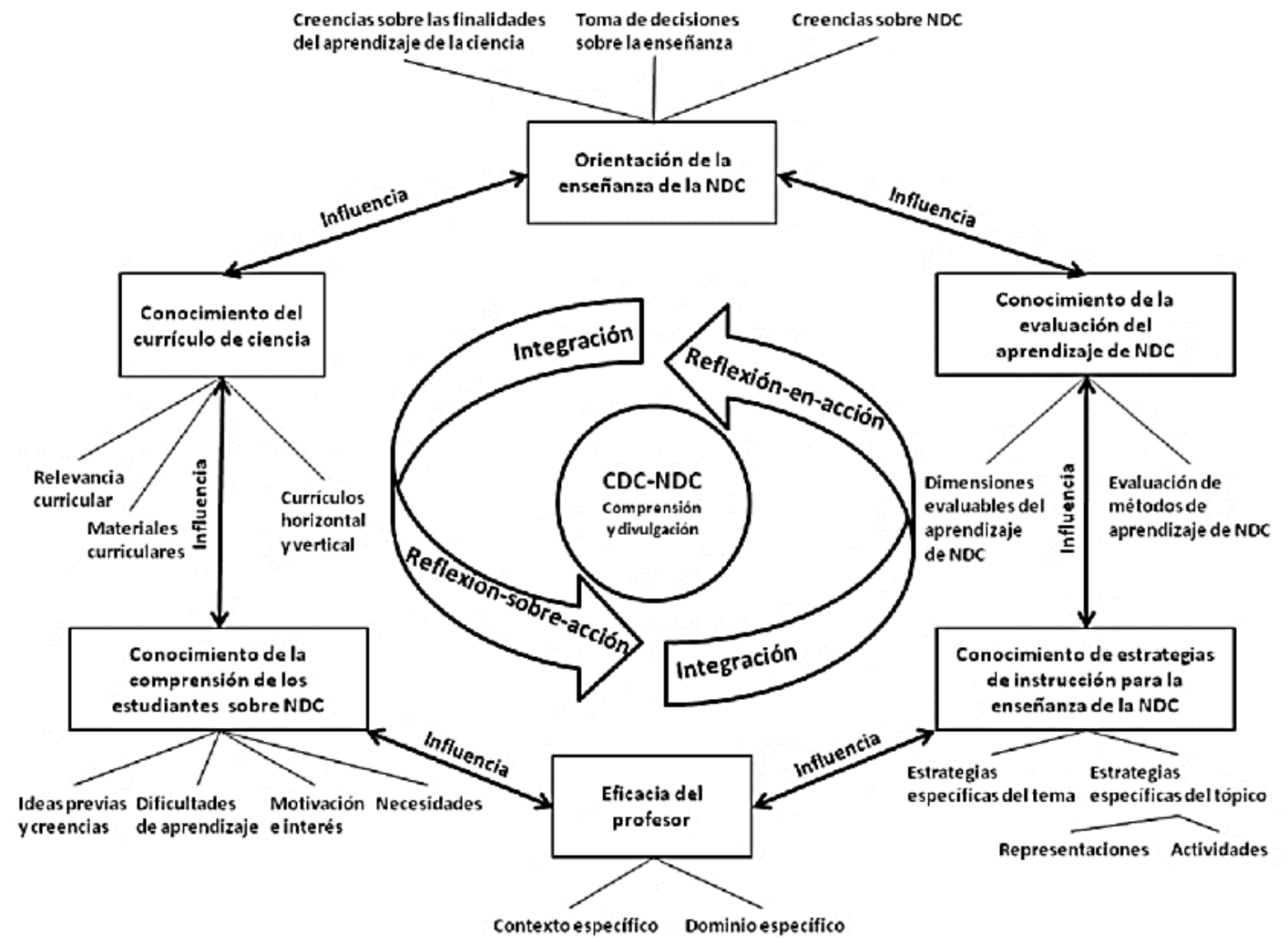

Figura 1. Modelo hexagonal de conocimiento didáctico del contenido para enseñanza de las ciencias (Park y Oliver 2008).

Para estos autores, el cDc es entendido como la comprensión y las actitudes de los profesores al momento de ayudar a un grupo determinado de alumnos para comprender un contenido específico a través de múltiples estrategias educativas y evaluaciones (Katzaroff, 2014), y estaría influenciado por: 1) las orientaciones para la enseñanza de las ciencias, 2) conocimiento acerca del currículo de las ciencias, 3) conocimiento sobre las comprensiones de los estudiantes acerca de las ciencias, 4) la eficacia del profesor, 5) el conocimiento sobre las instrucciones para la enseñanza de las ciencias y 6) un conocimiento sobre la evaluación del aprendizaje de los contenidos científicos.

Aunque, si bien, el primer componente se ha considerado algo ambiguo (Abell, Park, Hanuscin, Lee y Gagnon, 2009) debido a una definición inicial que considera al docente como partícipe dentro del proceso de educación científica y una segunda fase, centrada en una visión general de la educación científica que omite el papel del docente. 
No se puede olvidar que estas orientaciones influyen en gran medida en la construcción del CDC, ya que este se estructura como un mapa que guía las decisiones de la enseñanza, el uso de los materiales curriculares particulares, y las estrategias de instrucción y de evaluación. Por otra parte, la perspectiva epistemológica que el profesor posee sobre su disciplina científica tiene gran influencia en la toma de decisiones curriculares, la elección de los contenidos a enseñar, el diseño de sus actividades y su forma de evaluar (Adúriz-Bravo, 2005b; Colombo, 2004; Gil, 1993; Jiménez, 2003; Mellado, 1993; de Pro, 2003; Zambrano, 2003).

Hablar de un conocimiento sobre currículo en ciencias, implica hablar de un conocimiento sobre las relaciones entre los conceptos, tópicos y temas del currículo vigente, tanto desde los aspectos verticales como horizontales (Katzaroff, 2014). De igual forma, de los contenidos interdisciplinares y las estructuras y organizaciones dadas por los ministerios de educación, que para el caso colombiano serían: los Lineamientos curriculares (MEN, 1998), los Estándares básicos de competencias en ciencias naturales (MEN, 2004), y los Derechos básicos de aprendizaje (MEN, 2016), las cuales se convierten en imposiciones externas y pueden tener un efecto negativo en la reflexión de los docentes, así como una construcción de un CDC.

Lo anterior muestra una relación entre el conocimiento, su naturaleza, su legitimidad científica, su selección y organización para la enseñanza; todos estos aspectos representan un interés por el conocimiento científico que debe ser abordado de forma educativa, lo cual introduce un problema de orden epistemológico en un contexto educativo (Rengifo y Zambrano, 2009). Al resolver esta cuestión se logra identificar qué se puede enseñar de una disciplina y cómo decidir la organización del conocimiento para enseñar. Al mismo tiempo, pone de manifiesto la importancia de reconocer las estructuras sustantivas y sintácticas del contenido científico.

\section{Implicaciones del CDC-PCK para la enseñanza de la biología}

Es tan relevante la identificación de estos componentes y su integración en la construcción del CDC, que en los últimos años se han desarrollado un gran número de investigaciones en el campo de la didáctica de las ciencias naturales, tanto de sus aspectos generales, como en temas o tópicos específicos, y se han logrado establecer CDC específicos (Mavhunga, 2012). De esta manera, es común hallar trabajos que estudian la construcción de un Conocimiento Didáctico del Contenido Biológico (Chapoo, Thathong y Halim, 2014; Fonseca, 2017b; Valbuena, 2007; Valbuena et ál., 2009). Al mismo tiempo, se establecen conocimientos aún más específicos, como son los trabajos de Fonseca (2011), sobre el conocimiento didáctico del contenido del concepto de biodiversidad o los trabajos sobre conocimiento didáctico del contenido de célula (Cohen y Yarden, 2009; Katzaroff, 2014; Ravanal y López-Cortés, 2016; Yañez y Tapia, 2015).

Es desde estas investigaciones que la construcción de un CDC específico como conocimiento base para la enseñanza de conceptos como el de célula cobra gran importancia, ya que este tema emplea muchos conceptos abstractos, la mayoría de ellos de difícil asimilación para el alumno y, por tanto, necesita de una especial atención, reflexión y transformación didáctica. Además, el concepto de célula es fundamental en la organización de los saberes biológicos ya que, sin la comprensión de su estructura y función, los demás conceptos en Biología se vuelven incompletos o fragmentados (Katzaroff, 2014). Dicho de otra manera, el concepto de célula es un elemento clave, pues se presenta como una parte del contenido estructural de toda la biología.

Sumado a lo anterior, se encuentra la situación ampliamente conocida dentro de los espacios educativos formales y no formales, que es el hecho de que quien enseña ciencias en muchos casos no es un profesional formado en aspectos pedagógicos, sino, por el contrario, un profesional del área. Esto tiene como consecuencia que los procesos de enseñanza sean atravesados por una visión conceptual centrada en el contenido que, sin duda, tendrá implicaciones en el quehacer docente y en la manera como este lleva a cabo su labor. Así, el objetivo de este trabajo es determinar el conocimiento didáctico del contenido de un profesor de biología, la forma como este construye y utiliza este conocimiento en torno al concepto de célula y sus implicaciones para la enseñanza de la biología; teniendo como referencia las categorías del modelo hexagonal de Park y Oliver (2008).

\section{Metodología}

Este trabajo se desarrolló a partir de un paradigma interpretativo, que considera la existencia de realidades múltiples, construidas por los propios actores, desde un enfoque cualitativo (Creswell, 2010; Gatti y André, 2010), pues busca una comprensión e interpretación de las realidades presentes en la acción docente y, que al final, se desenvuelve siguiendo una metodología de estudio denominado caso único, según Stake (1999). Para esto se analizó la práctica de un docente de biología ${ }^{4}$, que se desempeña

4 Se usa la palabra docente dada su recurrencia e importancia en el contenido del trabajo, sin embargo, se aclara que el participante es un profesional en biología. Es decir, un biólogo que carece de formación pedagógica inicial. 
como profesor de ciencias naturales en grado sexto con cinco años de experiencia en un colegio de carácter privado de la ciudad de Tunja, Boyacá. El proceso de levantamiento de la información se llevó a cabo a partir de una triangulación metodológica entre observación no participante de clases, una entrevista estructurada, basada en el cuestionario de representación del contenido (ReCo) propuesto por Loughran, Mulhall y Berry (2004) -el cual consta de ocho preguntas que permiten indagar el conocimiento didáctico sobre las ideas centrales en torno al concepto de célula-y una interpretación de planes de área y planes de clase.

Para el análisis de los datos obtenidos mediante la entrevista por ReCo y las clases observadas, se utilizó el programa Atlas.ti ${ }^{\oplus}$ versión 6.2, y se implementaron dos niveles de análisis, el primero textual y el segundo conceptual. Por su parte, para el análisis de los documentos de clase proporcionados por el docente, se tomaron como referencia los Lineamientos curriculares en ciencias naturales del Ministerio de Educación Nacional (MEN, 1998), los Estándares básicos en competencias en ciencias natura- les (MEN, 2004) y los Derechos básicos de aprendizaje (MEN, 2016), los cuales pretenden que las generaciones que se están formando no se limiten a acumular conocimientos, sino que aprendan lo que es pertinente para su vida y puedan aplicarlo para solucionar problemas nuevos en situaciones cotidianas.

\section{Resultados y discusión}

Con los datos obtenidos en las observaciones de clase, fueron elaboradas unidades de registro a partir de la relectura de los documentos transcritos y se llevó a cabo una codificación teniendo en cuenta categorías deductivas, las cuales fueron tomadas del modelo hexagonal de Park y Oliver (2008). A partir de esto se pudo evidenciar que, en la acción del docente predominan de forma implícita aspectos relacionados con: el conocimiento sobre las orientaciones para la enseñanza de las ciencias, el conocimiento sobre el currículo de las ciencias y el conocimiento de la evaluación del aprendizaje de las ciencias, como se puede evidenciar en la figura 2.

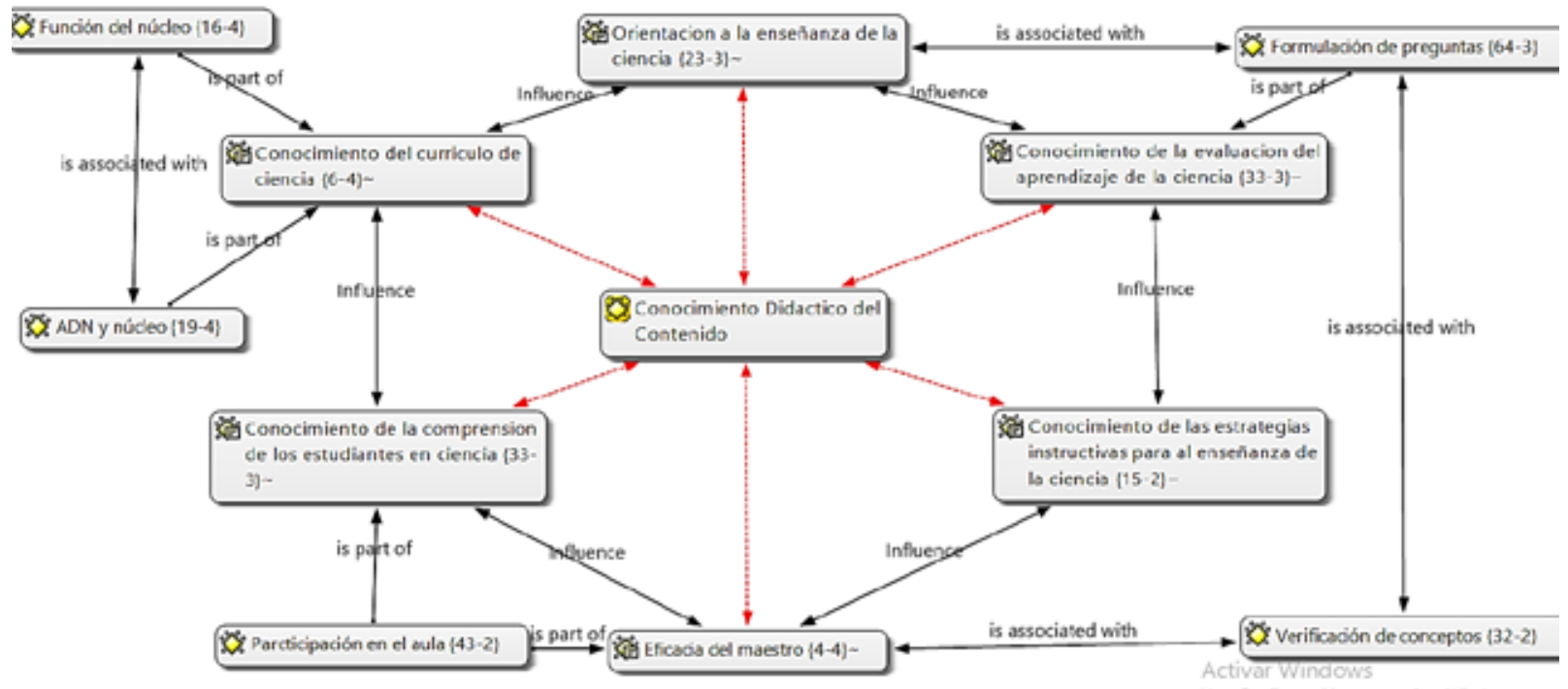

Figura 2. Red semántica del CDC del desarrollo de clase. Fuente: elaboración propia.

Desde el análisis se evidencia que el cDc del profesor está fuertemente influenciado por aspectos conceptuales propios de la disciplina, en este caso relacionados con las funciones del núcleo y su relación estrecha con el ADN, los cuales se soportan en el gran número de citas relacionadas entre estos conceptos (35), además de esto, fue posible percibir una reiterada formulación de preguntas cuyo objetivo es evaluar la memorización de un contenido específico. A continuación, se presentan los resultados y discusión de manera detallada de los tres componentes que predominaron en el análisis inicial. 


\section{Orientaciones hacia la enseñanza de las ciencias, conocimiento de la evaluación y conocimiento del currículo de las ciencias sobre el concepto de célula}

Si se parte del hecho de que los propósitos, contenidos, metodologías y evaluación de la enseñanza de la Ciencia están influenciados por el enfoque que se tenga al enseñar (Magnusson et ál., 1999) y por la propia concepción del docente, las orientaciones de la enseñanza de la Ciencia constituyen el referente conceptual en la toma de decisiones curriculares y metodológicas. Por esto, lo que enseñe un profesor, la manera como lo enseñe y cómo lo evalúe diferirá dependiendo de la visión que tenga acerca de cómo debe ocurrir el proceso, ya sea que defienda una posición transmisiva o, por el contrario, defienda otras perspectivas.

Con base en lo anterior, en algunos apartes de las clases orientadas por el docente es posible inferir una tendencia que centra la enseñanza de las ciencias -en este caso del concepto de célula- en una inalterada transferencia de información que limita la reflexión didáctica a aspectos de evaluación, que a su vez se reduce a la retención de ideas expresadas en respuestas y definiciones cortas dadas por los estudiantes. Esto se evidencia en códigos como Formulación de preguntas y verificación de conceptos, que en la práctica del docente están direccionadas a evaluar un contenido memorístico, en otras palabras, se encarga de transmitir a sus estudiantes conceptos inalterables sobre la célula, que se han configurado como verdades indiscutibles dentro de la biología (Carvalho, El-Hani y Nunes-Neto, 2017).

Aunado a esto, se hace evidente que, en la planeación el profesor se centra en el contenido, debido tal vez a su formación inicial o a una fuerte influencia del currículo nacional, lo pone de manifiesto el problema de la reflexión didacta, en términos de la selección de contenidos para enseñar, la organización y su evaluación. Estas son algunas acciones que limitan y dificultan la enseñanza de las ciencias naturales.

En contraposición, y desde algunos postulados teóricos (El-Hani y Mortimer 2007; Unesco, 2009), se establece que el aprendizaje de las ciencias debe proponer el desarrollo de un pensamiento lógico, facilitar una aproximación a conocimientos que la comunidad científica reconoce como válidos en un momento histórico dado o promover el desarrollo progresivo de estructuras conceptuales cada vez más complejas, que permitan una mejor comprensión de los conceptos científicos. De esta manera, queda en evidencia que la enseñanza de las ciencias no puede tener como objetivo la simple transmisión de conceptos
- leyes científicas que siga una metodología tradicional, donde la enseñanza y el aprendizaje se reduzcan a la memorización de conceptos, hechos, leyes, fórmulas y ejercicios logrando una "educación" en la que el alumno tiene su cabeza repleta de conocimientos aislados y no se logra desarrollar su espíritu comprensivo, reflexivo e innovador (Tacca, 2010).

De la misma manera, si partimos de lo dicho por Kind (2009), quien considera que los objetivos establecidos por el maestro definen en gran medida su conocimiento de las orientaciones para la enseñanza, podemos observar que en los documentos utilizados por el docente para guiar su área y su clase se trazaron como objetivos "Comprender el funcionamiento y estructura del núcleo celular" e "Identificar las partes del núcleo y sus componentes". Esto denota, de nuevo, concepciones de una biología centrada en los conceptos, en la cual el docente se dedica a transmitir una ciencia acumulativa; presentada como un cuerpo comprobado de conocimientos (De Pro, 2003).

Lo anterior puede verse reflejado en la figura 3, la cual muestra cómo el desarrollo de las clases se centra en la enumeración y explicación de conceptos centrales. Si bien es cierto que, para el aprendizaje de la biología es fundamental el reconocimiento de términos como el de célula y su estructura y función son importantes para la comprensión y articulación de la biología escolar (Katzaroff, 2014; Rodríguez, 2002), no podemos obviar el hecho de que únicamente un buen dominio del contenido específico garantizará el aprendizaje de este (Grossman et ál., 2005). Es necesario introducir aspectos contextuales que se aproximen a las realidades propias tanto de la biología, su implicación en la sociedad como de la experiencia cercana de cada estudiante. 


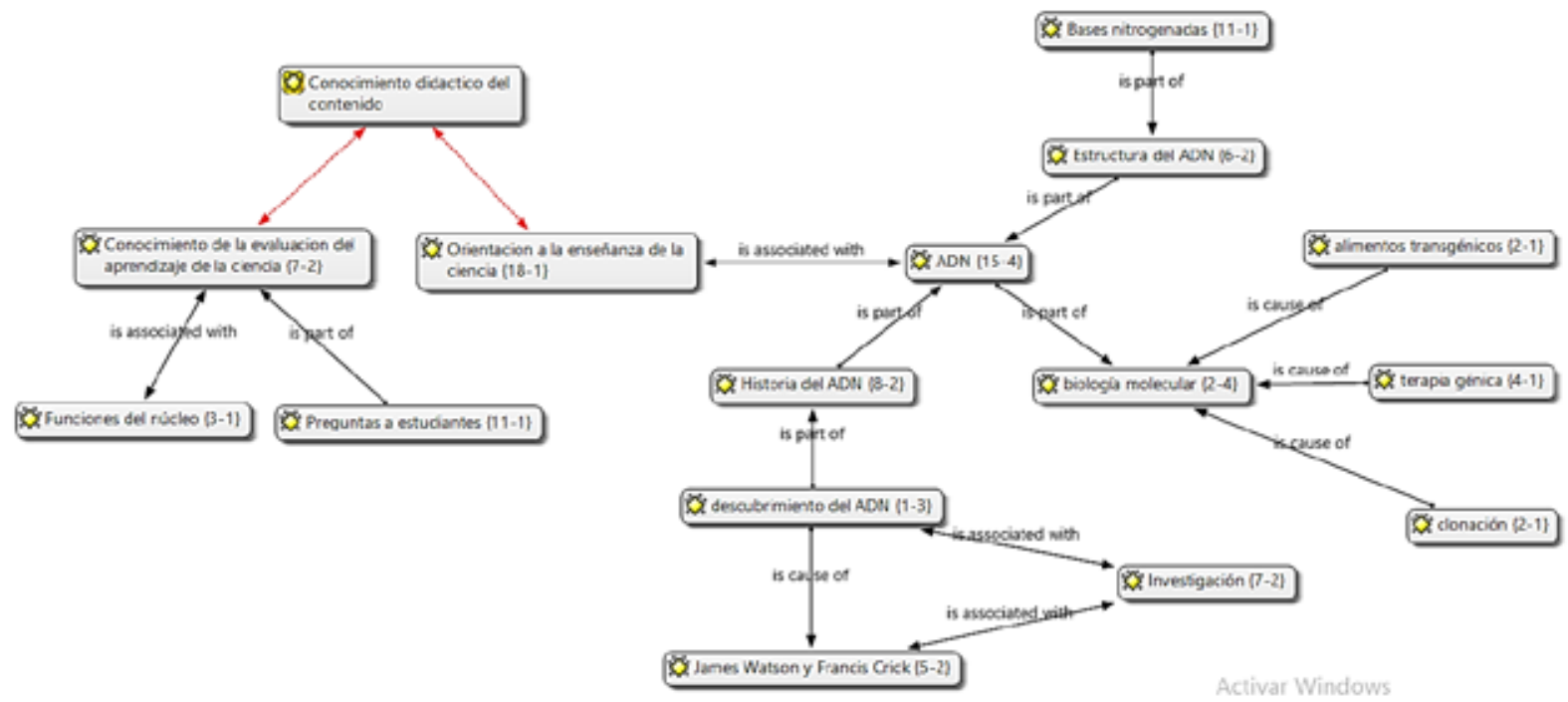

Figura 3. Red semántica sobre orientación a la enseñanza de las ciencias.

Fuente: elaboración propia.

Otro aspecto que se relaciona con el desarrollo de la clase y la carga conceptual de la misma frente al tema son las explicaciones que proporciona el docente, las cuales se podrían caracterizar como una de las formas que asume la enseñanza en muchas configuraciones didácticas (Litwin, 1997), el docente explica esperando que sus estudiantes aprendan (Ogborn, Kress, Martins, y McGillicuddy, 1996). Dentro de este proceso se espera que se dé lugar a una serie de relatos breves a los cuales podría atribuírseles dos momentos: el primero, una contextualización inicial del contenido y el segundo, brindar un espacio en el que se plantean una serie de preguntas y tareas que el estudiante resuelve y el profesor evalúa (Eder y Adúriz-Bravo, 2008). Aunque el docente dentro del desarrollo de su labor crea contextualizaciones y plantea preguntas, en muchas ocasiones se generan discursos prolongados y se deja de lado lo que caracteriza las explicaciones las cuales, según estos autores, deben ser breves monólogos del profesor, extraordinariamente articulados y que clarifican ideas que se consideran en particular relevantes, evaluando el proceso sobre la marcha.

En otro orden de ideas, se considera que una comprensión completa de la biología escolar requiere el estudio de la historia y epistemología de esta (Allchin, 2015; Rodríguez, 2000). Así, la enseñanza y aprendizaje de la célula como concepto científico y escolar no se realiza de forma descontextualizada o ahistórica, sino que debe promover que el estudiantado comprenda que el conocimiento científico es una creación humana y, como tal, está sujeta al contexto social, histórico y cultural en el que se encuentra inmerso el científico (Camacho 2010; Matthews 2014). Esto contrasta con los planteamientos del docente al decir:
Pues historia y epistemología no me centro, sé que hay aspectos importantes como los aportes dados por Antón van Leeuwenhoek y Brown, pero a decir verdad no me centro en estos campos... (Comunicación Personal. Respuesta frente al cuestionario ReCo, 20 de abril de 2018).

Aquí es evidente que para el docente la naturaleza e historia de su disciplina, y del concepto enseñado, pasan a un segundo plano en la planeación, aunque en el desarrollo de sus clases retoma elementos de James Watson y Francis Crick, como actores fundamentales en el desarrollo histórico del contenido (Véase figura 3). Sin embargo, solo los menciona, no hace énfasis en los procesos e implicaciones de sus descubrimientos, ni cómo estos fundaron uno de los "paradigmas" modernos de la biología. Lo mismo sucede con menciones de conceptos como clonación, terapia génica y alimentos transgénicos que, como se evidencia en la red semántica sobre orientación a la enseñanza de las ciencias, son referencias superfluas y sin fundamento epistemológico.

Desde esta perspectiva (historia y epistemología de la ciencia), un análisis profundo de la disciplina ayuda a identificar qué se puede enseñar de ella y cómo decidir la organización del conocimiento que se enseñará (Rengifo y Zambrano, 2009), es decir, influye en las decisiones curriculares que el docente toma. Sin embargo, historia y filosofía de las ciencias no son suficientes para esto, el docente debe recurrir tanto a aspectos propios de las disciplinas como las estructuras sintácticas y sustantivas, como a disciplinas auxiliares como la pedagogía, la psicología, la sociología, la antropología, la lingüística y la ética (Duit, 2006) que, a su vez, influirían en la construcción del CDC. 
Adicionalmente, los saberes curriculares que hacen referencia a los contenidos, objetivos y métodos, (mediante los cuales el docente orienta el contenido que enseña), se esquematizan a partir de estructuras curriculares verticales y horizontales (Grossman, 1990). Se evidencia que el docente organiza el contenido de manera lineal y sigue las directrices de los estándares básicos de competencias, a partir de una estructuración de los núcleos conceptuales del tema. Más adelante, establece el desarrollo siguiendo "protocolos" rígidos y estructurados, al plantear sus clases de la siguiente manera: "1) la célula y sus funciones; 2) descubrimiento de la célula; 3) características de la célula; 4) funciones de la célula", así, el docente se traza un plan horizontal para el contenido que va a enseñar.

Los lineamientos curriculares estipulados por el MEN son cartas de navegación que usan los docentes para la planeación de sus clases y que se pueden considerar como un sistema flexible para la organización de un contenido específico y con unos objetivos determinados. En la planeación del docente, es evidente la formulación de un objetivo particular, sin embargo, no es pertinente la organización ni el desarrollo de los contenidos para lograrlo, ya que, en primera medida, no hay una secuencia lógica que oriente el contenido; inicia en la célula y sus funciones pasa por algunos elementos históricos de descubrimiento de la célula, aun cuando él considera que no son relevantes en su enseñanza, y finaliza redundando en funciones de la célula.

El desarrollo de este currículo horizontal, también se realiza a partir de la organización de conceptos auxiliares relacionados con cada contenido, de esta forma la figura 4 muestra cómo se estructuran los conceptos claves de un tema específico trabajado por el docente, en este caso las funciones del núcleo. Sin embargo, el docente centra su explicación en enumerar las partes del núcleo y la relación de estas con el material genético, dejando implícito el verdadero objetivo de su organización.

De nuevo, pero ahora desde la selección y organización de los contenidos, es evidente la importancia que se da a los aspectos conceptuales y teóricos. Se asumen con mayor legitimidad los saberes de orden teórico-conceptual, pues se presupone que estos hacen referencia a contenidos propios de las ciencias exactas, la biología incluida. De hecho, ni siquiera se hacen distinciones de los diferentes niveles de abstracción de estos saberes (Casarini, 2013).

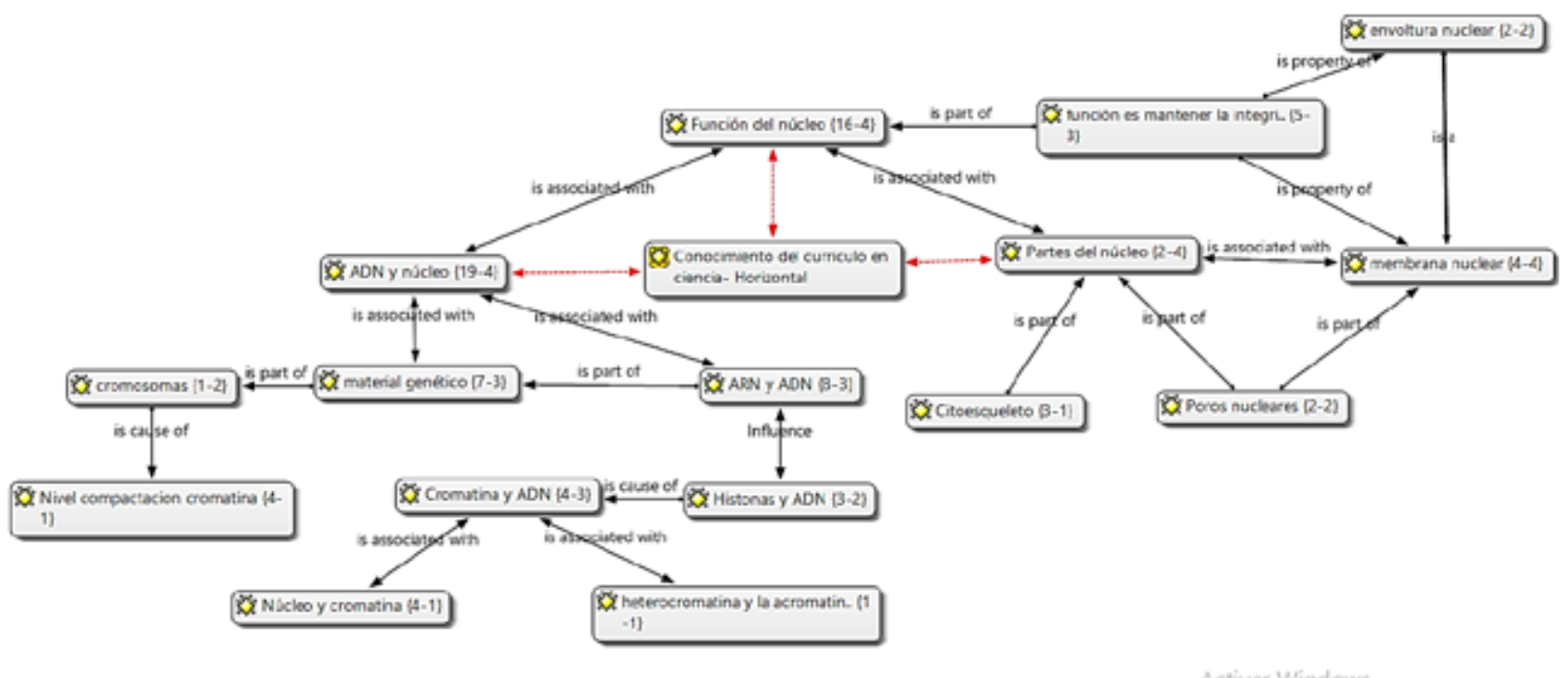

Figura 4. Red semántica sobre el conocimiento del currículo en ciencias.

Fuente: elaboración propia.

De vuelta a la figura tres, es visible que hay una inclusión de elementos adicionales a la estructura clásica del currículo horizontal seguido por el docente, esto es, algunas menciones de aspectos relativamente nuevos en investigaciones sobre biología molecular, alimentos transgénicos, terapia génica y clonación, el último de estos es tratado en algunos libros de texto, y con escasas menciones por los lineamientos curriculares colombianos (MEN, 1998; MEN, 2004; MEN, 2016). Pero a pesar de este intento de integración de aspectos vinculados a estructuras sustantivas del contenido biológico, su relación con la clase es mínima, y solo se hace una referencia que deja de lado aspectos contextuales de estos. Así, se deja una marcada evidencia de una influencia relativa del currículo nacional 
sobre la selección de dichas estructuras sustantivas, ya que el profesor no modifica los planes y programas de estudio establecidos por el ministerio (Ravanal y López-Cortés, 2016).

\section{Consideraciones finales}

Cada uno de los instrumentos analizados en el marco del estudio (revisión de planeación de clase, entrevista y observación de clase) debe actuar como parte de un sistema que centre su funcionalidad en una continua armonía para beneficiar el aprendizaje. Pues lo que se planea, lo que se piensa y lo que se hace en el aula de clase determinan en gran medida un CDC orientado hacia la potenciación de la enseñanza de las ciencias.

Se denota una fuerte influencia del currículo en la estructura sustantiva del contenido para enseñar, esta, predeterminada en gran medida por los planes de asignatura (desligados de los estándares y lineamientos curriculares del Ministerio de Educación Nacional), los cuales usa el docente como base para su planeación de clase y que, a pesar de modificar e intentar vincular actividades que enriquezcan su labor. En ocasiones carece de una aplicabilidad desde la orientación hacia la enseñanza de las ciencias; esto se puede generar debido a la presión que ejerce la unidad educativa sobre el cumplimiento de dicho currículo en la institución.

Los maestros deben tomar decisiones para el proceso de enseñanza que van a desarrollar, la mayoría de estas se insertan en decisiones de orden curricular, las cuales se intentan integrar a un contexto de enseñanza determinado y unas nociones conceptuales dadas por otros. Al hacerlo, el profesor está estrictamente ligado al desarrollo curricular, pues está determinando todas las fases del proceso de instrucción, como la organización de los contenidos, la generación de estrategias de enseñanza, el diagnóstico de problemas y la búsqueda de soluciones que regulan la práctica. Por tal razón, es necesario que las reflexiones curriculares se basen en aspectos didácticos y epistemológicos de cada disciplina y que fundamenten de forma coherente la selección, organización y desarrollo de los contenidos que se enseñarán.

\section{Referencias}

Abell, S. K., Park, M. A., Hanuscin, D. L., Lee, M. H. y Gagnon, M. J. (2009). Preparing the next generation of science teacher educators: A model for developing Pck for teaching science teachers. Journal of Science Teacher Education, 20(1), 77-93.

Adúriz-Bravo, A. (2005a). ¿Qué naturaleza de la ciencia hemos de saber los profesores de ciencias? Una cuestión actual de la investigación didáctica. Tecné, Episteme y Didaxis: TED (Número Extraordinario), 23-33.

Adúriz-Bravo, A. (2005b). Una introducción a la naturaleza de la ciencia. La epistemología en la enseñanza de las ciencias naturales. Bogotá: Fondo de Cultura Económica.

Allchin, D. (2015). Hooks, Lines and Sinkers. The American Biology Teacher Journal,77 (9), 718-720.

Bolívar, A. (2005). Conocimiento Didáctico del Contenido y didácticas específicas. Profesorado. Revista de currículum y formación del profesorado, 9(2), $1-25$.

Camacho, J. (2010). Concepciones del profesorado y promoción de la explicación científica en la actividad química escolar. Aportes de un modelo de intervención desde la Historia de la Ciencia para la enseñanza de la electroquímica [Tesis de doctorado, Pontificia Universidad Católica de Chile]. Repositorio institucional UC. http://www7.uc.cl/ sw_educ/educacion/grecia/plano/html/pdfs/biblioteca/DOCTOR/TesisDoctJohCa.pdf

Campos, J. (2016). Conocimiento profesional de los profesores: formación de profesores en dos agrupamientos de escuelas. Revista Electrónica Interuniversitaria de Formación del Profesorado, 19 (2), 273-287. https://doi.org/10.6018/ reifop.19.2.255681

Can, Ş., Doğru, S. y Bayir, G. (2017). Determination of pre-service classroom teachers Technological Pedagogical Content Knowledge. Journal of Education and Training Studies, 5 (2), 160-166. https:// doi.org/10.11114/jets.v5i2.2083

Carlsen, W. (1999). Domains of teacher knowledge. En J. Gess-Newsome y N. Lederman (Eds.), Examining pedagogical content knowledge (pp. 133-144). Dordrecht: Kluwer Academic Publishers Londres: Board. 
Carvalho, I. N., El-Hani, C. N. y Nunes-Neto, N. F. (2017). Conteúdos conceituais: reduzindo e reestruturando o currículo de Biologia para o ensino médio. Anais do xı Encontro Nacional de Pesquisa em Educação em Ciências - XI ENPEC. Universidade Federal de Santa Catarina, Florianópolis, sc.

Casarini, M. (2013). Teoría y diseño curricular. México: Trillas.

Chapoo, S., Thathong, K. y Halim, L. (2014). Understanding biology teacher's Pedagogical Content Knowledge for teaching "The Nature of Organism". Procedia-Social and Behavioral Sciences, 116, 464-471. https://doi.org/10.1016/j.sbspro.2014.01.241

Cohen, R. y Yarden, A. (2009). Experienced junior-high-school teachers' PCK in light of a curriculum change: "The cell is to be studied longitudinally". Research in Science Education, 39, 131-155.

Colombo, L. Y. (2004). ¿Es importante la epistemología de las ciencias en la formación de investigadores y de profesores en física? Enseñanza de las Ciencias, 22(3), 455-462. https://www.raco.cat/index.php/ Ensenanza/article/view/21994

Cossío, E. F. y Hernández, G. (2016). Las teorías implícitas de enseñanza y aprendizaje de profesores de primaria y sus prácticas docentes. Revista mexicana de investigación educativa, 21 (71), 1135-1164. http:// www.scielo.org.mx/scielo.php?script=sci_arttext\&pid=S1405-66662016000401135\&lng=es\&tlng=es

Creswell, J. (2010). "Mapeo del paisaje en desarrollo de la investigación de métodos mixtos" En A. Tashakkori y C. Teddlie (Eds.), Manual de Sage de Métodos mixtos en investigación social y del comportamiento (pp. 45-68). California: Sage.

de Jong, O. y Van der Valk, A. E. (2007). Science teachers' PCK and teaching practice: Learning to scaffold students' open-inquiry learning. En R. Pinto y D. Couso (Eds.), Contributions from Science Education Research (pp. 107-118). Springer, Dordrecht, The Netherlands. https://doi. org/10.1007/978-1-4020-5032-9_5

de Pro, A. (2003). La construcción del conocimiento científico y los contenidos de ciencias. En M. Jiménez, A. Caamaño, E. Pedrinaci y A. de Pro. Enseñar Ciencias (pp. 33-54). Barcelona: Editora Graó

Duit, R. (2006). La investigación sobre enseñanza de las ciencias. Un requisito imprescindible para mejorar la práctica educativa. Revista Mexicana de investigación educativa, 11(30), 741-770.
Eder, M. L. y Adúriz-Bravo, A. (2001). Aproximación epistemológica a las relaciones entre la didáctica de las ciencias naturales y la didáctica general. Tecné, Episteme y Didaxis:TEd, 9, 2-16. https://doi. org/10.17227/ted.num9-5618

El-Hani, C. N. y Mortimer, E. F. (2007). Multicultural education, pragmatism, and the goals of science teaching. Culture Studies of Science Education, 2 (3), 657-702. https://doi.org/10.1007/ s11422-007-9064-y

Fernández, C. (2014). Knowledge base for teaching and pedagogical content knowledge (PCK): Some useful models and implications for teachers' training. Problems of Education in the 21st Century, 60, 79-100.

Fernández, C. (2015). Revisitando a base de conhecimentos e o conhecimento pedagógico do conteúdo (PCK) de professores de ciência. Revista Ensaio, 17 (2), 500-528. https://doi. org/10.1590/1983-21172015170211

Fonseca, G. (2011). El Conocimiento Didáctico del Contenido del concepto de biodiversidad en profesores en formación de biología. Un estudio de caso desde el diseño de una unidad didáctica. Bio-grafía. Escritos sobre la biología y su enseñanza (Edición extraordinaria), 401-412.

Fonseca, G. (2017a). El conocimiento pedagógico del contenido en profesores de biología, una revisión documental. Bio-grafía. Escritos sobre la biología y su enseñanza, 10 (19). https://doi.org/10.17227/ bio-grafia.vol.10.num19-7219

Fonseca, G. (2017b). Aportes en la comprensión acerca de la construcción del conocimiento profesional del profesor de biología. Bio-grafía. Escritos sobre la Biología y su Enseñanza (Edición extraordinaria), 302-310. https://doi.org/10.17227/bio-grafia. extra2017-7119

Friedrichsen, P., Driel, J. H. V. y Abell, S. K. (2011). Taking a closer look at science teaching orientations. Science Education, 95 (2), 358-376. https://doi. org/10.1002/sce.20428

Gallego, A. P. y Gallego, R. (2006). Acerca de la didáctica de las ciencias de la naturaleza: Una disciplina conceptual y metodológicamente fundamentada. Bogotá: Magisterio. 
Gatti, B. A. y André, M. (2010). The relevance of qualitative research methods in education in Brazil. En R. Bohnsack, N. Pfaff y W. Weller (Eds.) Qualitative analysis and documentary method in international education research (pp. 41-52). Germany: Barbara Budrich Publishers.

Gess-Newsome, J. (1999). Pedagogical Content Knowledge: an introduction and orientation. En J. Gess-Newsome y N. Lederman (Eds.), Examining Pedagogical Content Knowledge (pp. 3-17). Dordrecht, The Netherlands: Kluwer Academic Publishers.

Gess-Newsome, J. y Carlson J. (2013). the Pck summit consensus model and definition of pedagogical content knowledge. En The Symposium "Reports from the Pedagogical Content Knowledge (PCK) Summit, ESERA Conference 2013, september.

Gil, D. (1993). Contribuciones de la historia y de la filosofía de las ciencias al desarrollo de un modelo de enseñanza/aprendizaje como investigación. Enseñanza de las Ciencias, 11 (2), 197-212.

Gómez, M. A. (2005). La transposición didáctica: historia de un concepto. Revista Latinoamericana de Estudios Educativos (Colombia), 1(1), 83-115.

Grossman, P. L. (1990). The making of a teacher: Teacher knowledge and teacher education. New York: Teachers College Press.

Grossman, P. L., Wilson, S. M. y Shulman, L. S. (2005). Profesores de sustancia: el conocimiento de la materia para la enseñanza. Profesorado. Revista de currículum y formación del profesorado, 9(2), 1-25.

Hashweh, M. Z. (2005). Teacher pedagogical constructions: a reconfiguration of pedagogical content knowledge. Teachers and Teaching: theory and practice, 11 (3), 273-292.

Hashweh, M. Z. (2013). Pedagogical Content Knowledge: Twenty-five years later. En C. Craig, P. Meijer y J. Broeckmans (Eds.), From teacher thinking to teachers and teaching: The evolution of a research community (Advances in Research on Teaching, Volume 19) (pp. 115 - 140). Emerald Group Publishing Limited.

Helmes, J. y Stokes, L. (2013). A meeting of minds around Pedagogical Content Knowledge: designing an international pck summit for professional, community, and field development. http://www.inverness-research.org/reports/2013-05_Rpt-PCK-Summit-Evalfinal_03-2013.pdf
Jiménez, M. (2003). La enseñanza y el aprendizaje de la biología. Enseñar Ciencias. Barcelona: Graó.

Katzaroff, J. (2014). Caracteristicas da base de conhecimentos de professores no ensino de biologia celular a partir de um curso de formação continuada. [Tesis de maestría, Universidade Estadual Paulista]. Repositorio institucional UNESP, https://repositorio.unesp.br/ handle/11449/116066

Kind, V. (2009). Conocimiento pedagógico del contenido en la enseñanza de las ciencias: perspectivas y potencial de progreso. Studies in Science Education, 45 (2), 169-204. https://doi. org/10.1080/03057260903142285

Lafrancesco, G. M. (2005). Didáctica de la Biología. Aportes a su desarrollo. Bogotá: Magisterio

Litwin, E. (1997). Las configuraciones didácticas. Una nueva agenda para la enseñanza superior. Buenos Aires: Paidós.

Loughran, J., Mulhall, P. y Berry, A. (2004). In search of pedagogical content knowledge in science: Developing ways of articulating and documenting professional practice. Journal of Research in Science Teaching, 41(4), 370-391.

Magnusson, S., Krajcik, J. y Borko, H. (1999). Nature, sources and development of pedagogical content knowledge for science teaching. En J. Gess-Newsome y N. Lederman (Eds.), Examining pedagogical content knowledge: the construct and its implications for science education (pp. 95-132). Dordrecht: Kluwer Academic Publishers.

Martínez, C. A. (2016). La investigación del conocimiento profesional del profesor(a) sobre el conocimiento escolar. En C. A. Martínez (Ed.), El conocimiento profesional del profesor(a) de ciencias de primaria sobre el conocimiento escolar: dos estudios de Caso, en Aulas Vivas y Aulas Hospitalarias del Distrito Capital de Bogotá (pp. 15-31). Bogotá, Colombia: Universidad Distrital Francisco José de Caldas.

Martínez, C. A. y Valbuena, É. O. (2013). Complejidad del conocimiento profesional de una profesora de ciencias de Primaria sobre el conocimiento escolar. Campo Abierto, 32 (1), 117-135. https://mascvuex.unex.es/revistas/index.php/campoabierto/ article/view/1391 
Matthews, M. R. (2014). The rapprochement between history, philosophy, and science education. En M. R. Matthews, Science Teaching: The contribution of History and Philosophy of Science. 20th Anniversary Revised and Expanded Edition (pp. 1-22). New York: Routledge.

Mavhunga, E. (2012). Explicit inclusion of topic specific knowledge for teaching and the development of $\mathrm{PcK}$ in pre-service science teachers. [Tesis de doctorado, Universidad de Witwatersrand]. Repositorio institucional WITS. http://hdl.handle. net/10539/15877

Mellado, V. (1993). Contribuciones de la filosofía de la ciencia a la didáctica de las ciencias. Enseñanza de las Ciencias, 11(3), 331-339.

Ministerio de Educación Nacional. (1998). Lineamientos Curriculares en Ciencias Naturales y Educación Ambiental. Bogotá: Magisterio. Ministerio de Educación Nacional.

Ministerio de Educación Nacional. (2004). Estándares Básicos de Competencias en Ciencias Naturales y Ciencias Sociales. Colombia: Ministerio de Educación Nacional.

Ministerio de Educación Nacional. (2016). Derechos Básicos de Aprendizaje. Ciencias Naturales. Colombia: Ministerio de Educación Nacional.

Ogborn, J., Kress, G., Martins, I. y McGillicuddy, K. (1996). Explaining Science in the Classroom, Buckingham: Open University Press.

Park, S. y Oliver, J. S. (2008). Revisiting the conceptualization of Pedagogical Content Knowledge (PCK): PCK as a conceptual tool to understand teachers as professional. Research in Science Education, 38, 261-284.

Park, S., Jang, J.-Y., Chen, Y.-C. y Jung, J. (2011). Is Pedagogical Content Knowledge (PCK) necessary for reformed science teaching? Evidence from an Empirical Study. Research in Science Education, 41, 245-260.

Pereira, J. M. (2010). Consideraciones básicas del pensamiento complejo de Edgar Morin, en la educación. Revista Electrónica Educare, 14(1), 42-58. https://doi.org/10.15359/ree.14-1.6

Porlán, R., Rivero, A. y Martín, R. (1997). Conocimiento profesional y epistemología de los profesores I: teoría, métodos e instrumentos. Enseñanza de las Ciencias, 15(2), 155-171.

Pozo, J. I. y Gómez, M. A. (2009). Aprender y enseñar ciencia: del conocimiento cotidiano al conocimiento científico. Madrid: Morata.
Ravanal, E. (2012). Creencias y práctica en profesores de ciencias: ideas para pensar un programa de desarrollo profesional desde la evaluación docente. Revista de Estudios y Experiencias en Educación, 11(22), 171-185.

Ravanal, E. y López-Cortés, F. (2016). Mapa del conocimiento didáctico y modelo didáctico en profesionales del área biológica sobre el contenido de célula. Revista Eureka sobre Enseñanza y Divulgación de las Ciencias, 13(3), 725-742. https://revistas.uca.es/index.php/eureka/article/ view/3001/2781

Rengifo, L. A. y Zambrano, A. C. (2009). La epistemología de Toulmin como referente para la selección de contenidos en la enseñanza de la biología. Revista Tecné, Episteme y Didaxis: TED, 26. https:// doi.org/10.17227/ted.num26-424

Rodríguez, M. L. (2000). Modelos mentales de célula: una aproximación a su tipificación con estudiantes de cou. [Tesis de doctorado, Universidad de La Laguna]. Repositorio institucional UL. http://riull. ull.es/xmlui/handle/915/9875

Rodríguez, M. L. (2002). La concepción científica de célula para la enseñanza de la biología: una reflexión aplicable a la escuela secundaria. Revista de Educación en Biología, 5(1) 41-50.

Rodríguez, B. y Martínez, L. F. (2016). Conocimiento profesional de profesores en ejercicio al abordar cuestiones sociocientíficas. Revista Tecné, Episteme y Didaxis: TED (Número Extraordinario), 14281434. https://revistas.pedagogica.edu.co/index. php/TED/article/view/4764

Shulman, L. S. (1986). Those who understand: Knowledge growth in teaching. Educational Research, 15 (2), 4-14. https://doi. org/10.3102/0013189X015002004

Shulman, L. S. (1987). Knowledge and Teaching: Foundations of the New Reform. Harvard Educational Review, 57(1), 1-23. https://doi.org/10.17763/ haer.57.1.j463w79r56455411

Stake, R. E. (1999). Investigación con estudio de caso. Madrid: Morata.

Tacca, D. (2010). La enseñanza de las ciencias naturales en educación básica. Investigación educativa, 14(26), 139-152. https://revistasinvestigacion.unmsm.edu.pe/index.php/educa/article/ view/4293/3429 
Unesco. (2009). Aportes para la enseñanza de las ciencias. Santiago de Chile: Laboratorio Latinoamericano de evaluación de la calidad de educación.

Valbuena, É. O. (2007). El conocimiento didáctico del contenido biológico: estudio de las concepciones disciplinares y didácticas de futuros docentes de la Universidad Pedagógica Nacional (Colombia) . [Tesis de doctorado, Universidad Complutense de Madrid]. Repositorio institucional, UCM. https:// eprints.ucm.es/7731/

Valbuena, É. O., Gutiérrez, A. M., Correa, M. A. y Amórtegui, E. F. (2009). Procesos formativos que favorecen la construcción del conocimiento profesional del profesor en futuros docentes de Biología. Revista Colombiana de Educación, 56, 156-179. https://doi.org/10.17227/01203916.7584
Yañez, R. y Tapia, G. (2015). El Conocimiento Didáctico sobre la célula y el análisis crítico de las propuestas (orientaciones) de enseñanza de dos profesionales del área biológica que ejercen en la educación secundaria. Bio-grafía. Escritos sobre la Biología y su Enseñanza (Edición extraordinaria), 1090-1101. https://doi.org/10.17227/20271034. vol.0num.0bio-grafia1090.1101

Zambrano, A. (2003). Cuestiones históricas y epistemológicas en torno a la enseñanza de las ciencias. En Formación del pensamiento científico. Cátedra Icfes Agustín Nieto Caballero.

Zambrano, A. (2005). Didáctica, pedagogía y saber. Bogotá: Magisterio. 